\title{
14. On the Vertical Displacement of the Sea Bottom in Sagami Bay Discovered after the Great Kwanto Earthquake of 1923.
}

\author{
By Torahiko TERADA, M.I.A. \\ Earthquake Research Institute, Tokyo Imperial University.
}

(Comm. 12 Feb., 1928.)

One of the most interesting results of investigations made in connection with the recent Great Kwanto Earthquake is the remarkable vertical displacement of the sea bottom in Sagami Bay which has been discovered by the extensive soundings carried out by the Hydrographic Office of the Navy. ${ }^{1)}$ Besides the question whether the displacement had been effected during the long interval of time between the two epochs of soundings, or mainly at the time of the earthquake, some skeptism seems to have been entertained as to the reality of the displacement, as the amount of the vertical movement was considerable, sometimes exceeding $100 \mathrm{~m}$., while the vertical change ascertained in the land area scarcely surpassed $2 \mathrm{~m}$. The present author shared the same doubt and carried out some investigations on this point, immediately after the publication of the result of soundings. He came to the conclusion $^{2}$ that the amounts of the displacements found cannot be plausibly explained by an inaccuracy in measurement alone and, therefore, could be accepted as real at least in the general feature of the results. As the report cited was too laconic on this point, omitting the statistical data, he may be excused to return to the question once more.

Among the possible errors of the deep sea sounding, the error due to waves is out of question in the present case, as it is limited to a few metres at most. The second source of error due to the inclination of the sounding wire may amount to a few per cents. This error will, however, increase proportionally with the depth. Thence, if this category of error is considerable, the apparent vertical displacement will show some statistical correlation with the depth measured. The third source of error which may sometimes become considerable is due to the error in the position of sounding. This is proportional to the tangent of the angle of slope, $\theta$, in the bed and may turn out very

1) The Report of E.I.C., 100 B (1925), 61.

2) Ibid., 63 (in Japanese). 
serious in the present case, as the slope is sometimes above 10 degrees. If this kind of error was considerable, the apparent displacement must show some statistical relation with the value of $\tan \theta$ of the corresponding spot.

To test these points, following procedures were taken. ${ }^{1)}$ First, the apparent vertical displacement was plotted against the initial depth as abscissa as shown in Fig. 1, in which each of the small dots represents a position of the bed, and the mark $\times$ gives the mean value of the displacements falling within an interval of the abscissa amounting to $100 \mathrm{~m}$. The interval is taken successively without overlapping and $\times$ marks the centre of gravity of all the points in the interval. It will be at once seen that there is no tendency of the larger displacements to be systematically clustered towards the greater depths. As to the mean values of the successive interval we may remark that the displacement is mostly negative above $1200 \mathrm{~m}$. depth, which may be of some importance, as it means that the deepest portion of the bed was depressed on average, in contrast to the coastal land area. On the other hand, the fact that the greatest displacements appear mostly in the middle depth may arouse the suspicion to the effect that they might have been affected by the third causes of error above mentioned. As regards this point, the readers are referred to Fig. 2., which represent the statistical relation between the vertical displacement, taken as the ordinate, and the quantity proportional to $\tan \theta$ of the spot, as the abscissa. For obtaining the latter quantity, the following method was adopted. A series of contour lines were drawn according to the depth given by the former soundings at an interval of $250 \mathrm{~m}$. depth. The orthogonal trajectory to these contour lines was then drawn passing through each point of observation. The length of the trajectory curve intercepted by the two consecutive contour lines between which the spot in question is situated, was measured and the reciprocal of the length was taken as a measure of slope which is proportional to $\tan \theta$. Since, however, the variation of slope was too remarkable for allowing us to attribute the value thus found immediately for all points within the interval, the slopes of the adjoining intervals were taken into account and the reasonable value for each spot was roughly determined by interpolation.

The figure will show that the greatest displacements are by no means confined to the region of the larger slope. In the figure,

1) The numerical calculations and plotting of diagrams were carried out by $\mathrm{Mr}$. K. Nisida, formerly Assistant in Aeron. Res. Inst., Tokyo Imp. Univ., to whom the present author wishes to expoess his best thanks. 
Fig. 1. The Relation of the Change of Depth with the Original Depth.

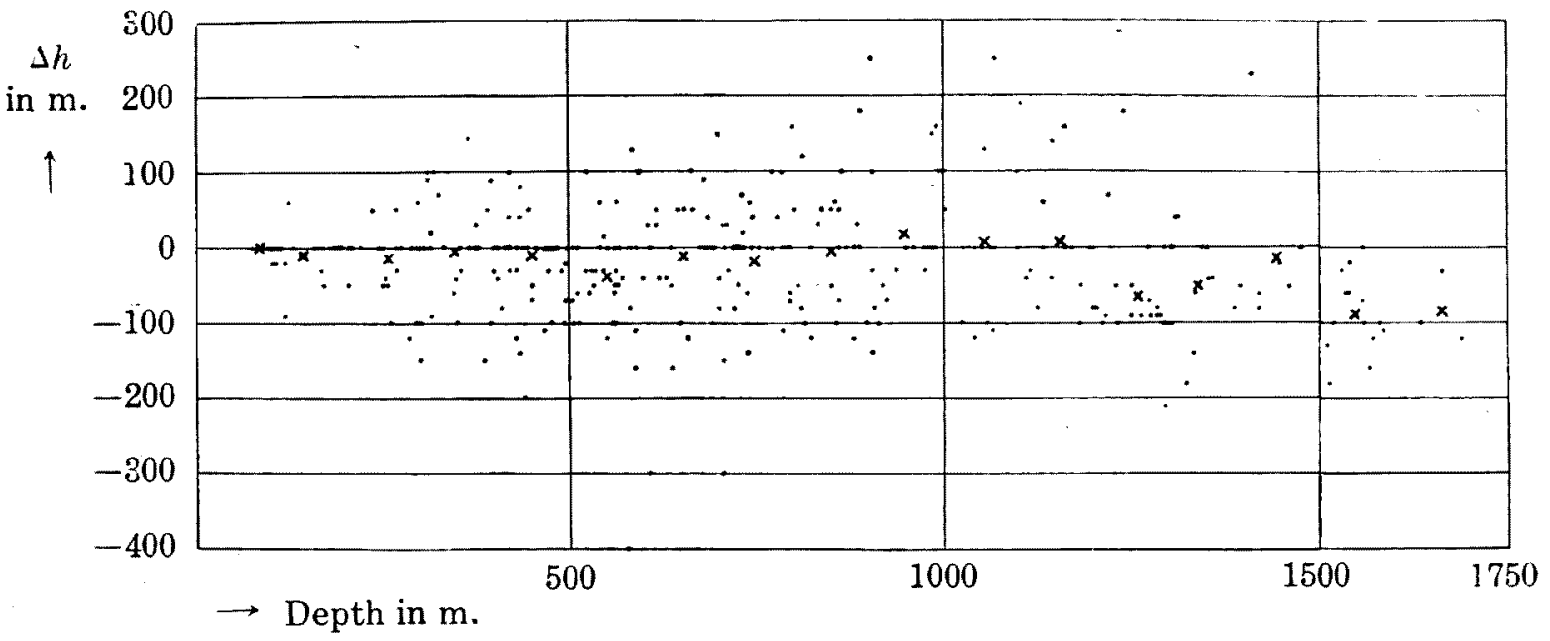

Fig. 2. The Relation of the Change of Depth with the Slope of the Bed.

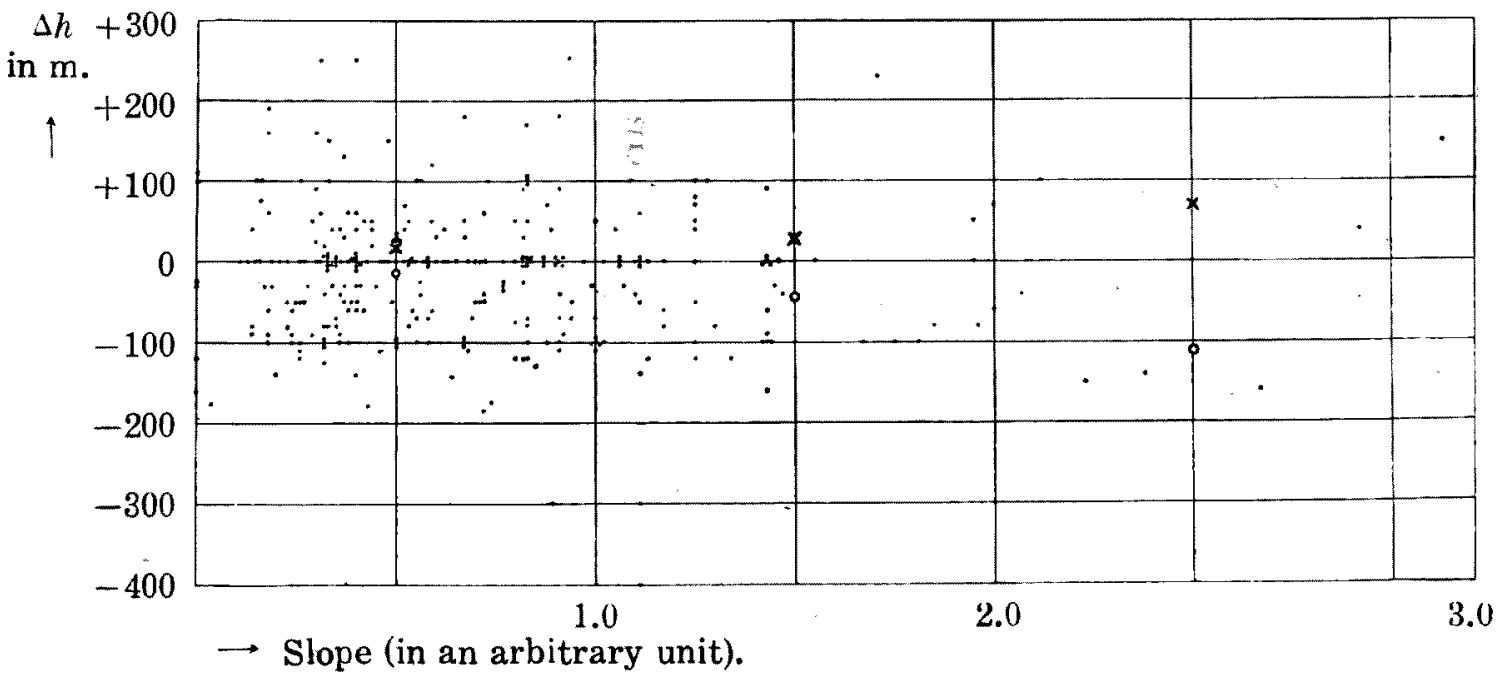


$x=1$ corresponds to $\tan \theta=0.155$ nearly; thence, in order that an error of $100 \mathrm{~m}$. may enter, the error in the position must amount to $645 \mathrm{~m}$. Such may well occur in the case of an inaccurate observation. Even if we admit that such be actually the case, it seems difficult to explain the characteristic mode of distribution of points in Fig. 2, unless we admit a highly incredible assumption that the determination of position is generally more inaccurate for the smaller slope.

The marks 0 and $x$ show the mean values of the positive and negative displacements respectively in the successive unit interval of $x$. The general increase of these mean values with the increase of $x$ will given an idea of the actual amount of the errors due to the very cause here concerned. Even if we discard all the points with $\tan \theta>1.0$, or even those $>0.5$, the number of points with $\Delta h$ of the order of $50 \mathrm{~m}$. is so great that to deny the reality of these values altogether may amount, in short, to totally abandonning the accepted method of sounding as incompetent.

Another point of support for the reality of phenomena may be afforded from a different side. If the recorded vertical movement was utterly illusory due to the inaccurate determination of position, it becomes incomprehensible how the displacement of one sign are distributed over a wide coherent area, while those with the opposite sign form another group with a considerable extent. The law of chance will evidently exclude such a state of things as highly improbable.

The indirect support will also be afforded by the accumulation of the facts from other quarters which appear causally connected with the observed vertical displacement. An example of these facts has already been given from the side of geological and morphological investigation. ${ }^{1)}$ Another example will be given in the paper by the author and Miyabe, which follows immediately. As all the arguments brought above are of passive nature, we must reserve some margin of possibility. It will, however, appear so far plausible to assume the validity of the general feature of the apparent vertical displacements, even if we exclude some extreme values as improbable.

1) For example see: N. Yamasaki, Rep. E.I.C., 100 B (1925), 11. 